\section{E-LOGOS}

ELECTRONIC JOURNAL FOR PHILOSOPHY ISSN 1211-0442

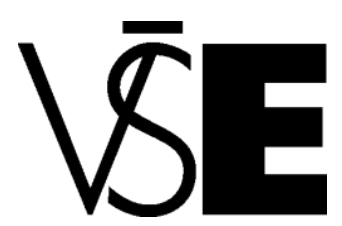

University of Economics

Prague

\title{
Concerning the Solution to the Russell's Paradox
}

I. M. R. Pinheiro

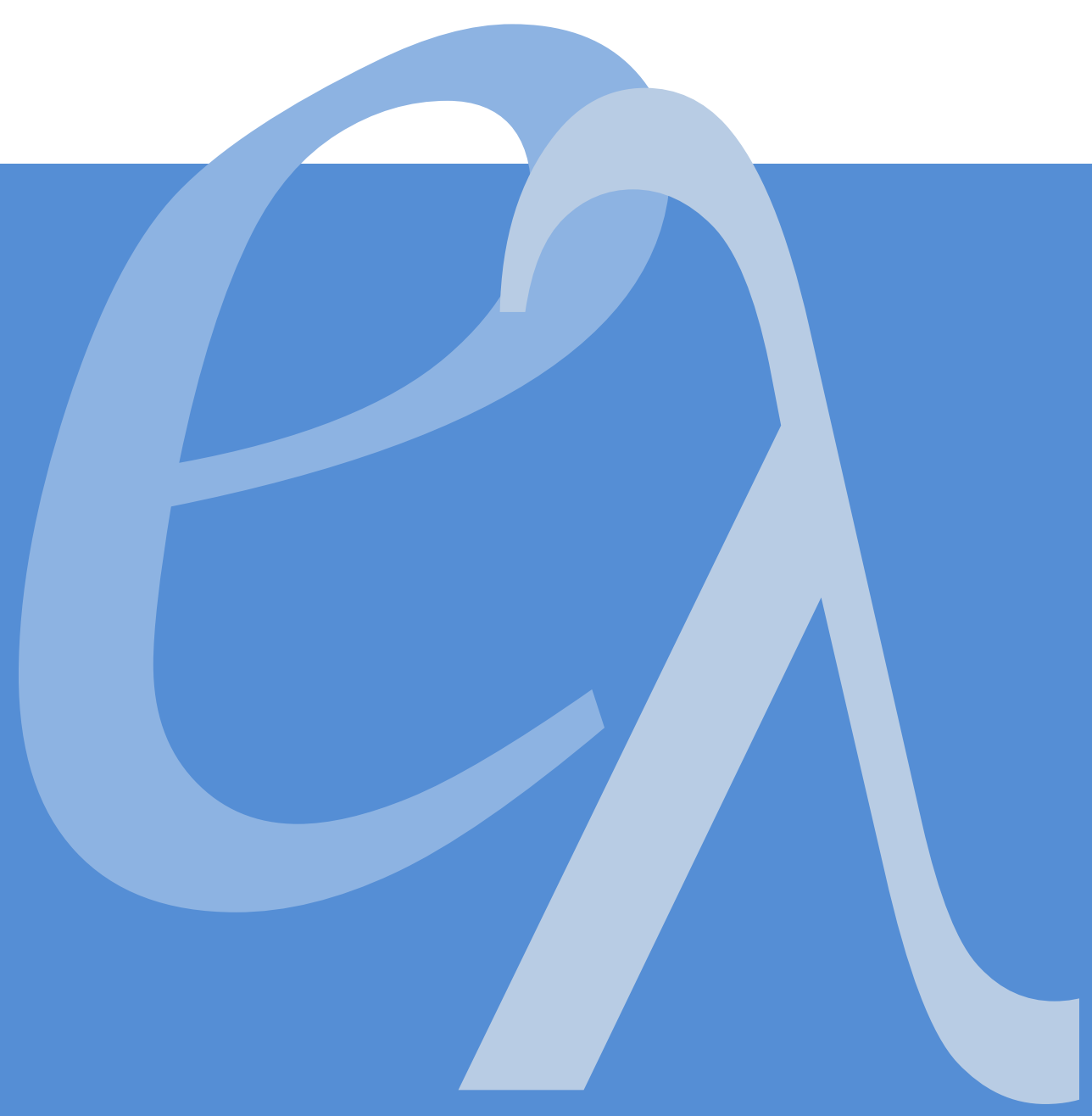




\section{Abstract}

In this note, we analyze and propose solution to the Russell's Paradox. We prove that the paradox is just an allurement to help us teach people the foundations of Mathematics properly.

Everything in the world, in general, is relative. It would be expected that things in Mathematics, an extreme reduction of the natural world, would be relative as well.

The secret, in dealing with 'Russell's Paradox', is acknowledging the relativism of the mathematical entities, especially the attachment of those to time constraints.

Keywords: Russell's Paradox, Russell, normal sets, inclusion, subset. 


\section{Introduction}

In [A. D. Irvine, 2009], we find out that Bertrand Russell ([A. D. Irvine, 2010]) wrote to Gottlob Frege about this paradox in June of 1902.

Frege would be working on the foundations of Arithmetic, printing his second book on the topic, when he received a letter from Russell letting him know about a possible inconsistency of weight in his theories (Frege's).

Russell basically had created a set that contained all sets that are not members of themselves and had called such a set $R$ (see [Wolfram, 1999]).

"Let $R$ be the set of all sets which are not members of themselves. Then $R$ is neither a member of itself nor not a member of itself. Symbolically, et $R=\{x: x \in x\}$. Then $R \in R$ iff $R \in R . "$

In the symbols above, $x$ stands for set $x$, as we know.

If we then make $x=R$ and forget what the letters refer to, we get that $R$ belongs to $R$ iff $R$ does not belong to $R$, which is what we read from the symbols at the end of the quote.

So, in seeing that definition, we could be wondering about whether $R$ would be itself inside of that set or not, since $R$ is itself a set.

To find out that, we would have to 'test the condition of pertinence to $R$ '. So, let's see: Does $R$ belong to $R$ ?

Then, thought Russell, 'I don't know', but if $R$ does belong to itself, then we have a problem because the condition would tell me that it cannot belong to itself, the condition of pertinence. On the other hand, if $R$ does not belong to itself, then the pertinence condition is satisfied, what then means that $R$ does belong to itself.

Then, thought Russell, I have just found a set that makes it impossible, that our theories be completely sound so far, since such a set could, by all the rules that we have created, exist. Yet, because of the same rules, it cannot exist, since assuming its existence leads us to a contradiction.

\section{The special set $R$, sometimes called 'normal set'}

Interesting that there are scientific texts calling the elements of $R$ 'normal sets' (see, for instance, p. 45 of [Stephan Körner, 1986]), meaning that people actually think that a set that does not belong to itself is more acceptable than a set that belongs to itself.

When we think of Geometry, this is not so acceptable, that a set belongs to itself, is it not?

Geometrically, any set could be put over itself without us being able to tell the difference between the first image of the set and the one that we have placed over it. 
We tend to think that, in this case, one set does not belong to the other, but that they are equal instead.

We tend to think that things that have other things are bigger than the things that belong to them like the jars that have, for instance, lollies. The lollies are inside of the jar, then the jar 'gives a home' to the lollies and the lollies belong to the jar... . That makes us think that one thing, $X$, can 'give a home' to another thing, $Y$, only if $X$ is bigger (in size) than $Y$.

If we think of the concrete world, we obviously cannot have a set belonging to itself, therefore, for it would be impossible to dress a can of juice, for instance, with another of the same dimensions without destroying the shape of the can, like no two things can occupy the same space, like precisely the same, and that we "know' 'for sure".

Mathematics, however, has taken off the dimensions of things.

In it, it is possible to deal with 'entities' inside of a sheet of paper: They become things that cannot be seen as anything that be not the own sheet of paper, that is, the entities 'are absorbed' by the object they lie over.

'No thing' is 'bidimensional' in our world, but things may be 'bidimensional' in Mathematics, such as the straight line in the Cartesian plane, therefore we cannot understand, if using our 'natural imagination' (call it 'the concrete world reasoning'), the objects in the Cartesian plane.

That was the problem Russell was having, trivially: These illusions, created by Mathematics, could make it possible that we had a set inside of itself.

Notice that we have a rule to build the set and a name for the set, but we do not have the set yet in the example of Russell.

The reason why $R$ could never have itself as an element in this situation is obvious: We will only have an $R$ after we have applied the 'pertinence condition' to all existing sets, and only to those. $R$ is not an existing set until we have finished applying the condition to all existing sets therefore $R$ itself should not be tested and can never be a member of itself.

On the other hand, if we write the rule with non-technical language, say $R$ is the set of sets that do not belong to themselves, then we could test $R$ in what regards the pertinence condition to the own $R$.

In this case, however, we are back to the paradox.

Notice that the conclusion of Russell was that such a set could not exist. Yet, he thought that, by all the existing rules, such a set should exist, like we were allowed to create it. 
Incredible enough, nobody would be able to exhibit a single set that has got itself as an element because, indeed, whatever has got something as an element should be bigger than that something, otherwise one thing would be equal to the other.

Such a set does not exist in reality, meaning in Mathematics. Therefore, the set of all sets that are elements of themselves is empty.

If we then create the supplementary set to this one, that which contains all sets that are not elements of themselves, such a set would have to contain all sets that there are in Mathematics, therefore would have to contain itself.

The secret to understand what is wrong with this 'paradox' is remembering that a set is a couple: (name of the set, elements of the set).

If we decide to call each set that we can create in Mathematics by a letter from our alphabet, we obviously will not have enough letters to name them. However, changing the rule for the assignment of names will not finish with 'the paradox'.

We obviously know, intuitively, that we cannot ever use one of the names that we have inside of the set to name it because they are 'different things', like one is part of the other together with other sets, say the 'lolly', whilst the other is the container, 'the jar'.

However, we have decided that every set 'can have a name'. Would that be the first 'impossible-to-be-named set'?

It does look like, in this case, naming the set is an impossibility... .

Perhaps we should then investigate the definition of set, as originally written by Peano since Russell himself, in his book ([B. Russell, 1903]), says, in section 67, that 'a class is a numerical conjunction of terms', that is, it is not a couple (name, elements), but simply (elements).

Of course, for us to have a 'paradox', we would need to have an axiom of creation of the entity 'set' stating that a set is a couple, (name, elements), not simply (elements).

Is it not easy to see that?

Interesting enough is the fact that [A. D. Irvine, 2009] tells us that

"...the principles governing set existence found within naive set theory, particularly the so-called Comprehension (or Abstraction) axiom. This axiom in effect states that any propositional function, $P(x)$, containing $x$ as a free variable can be used to determine a set. In other words, corresponding to every propositional function, $P(x)$, there will exist a set whose members are exactly those things, $x$, that have property $P . "$

what implies that there is an axiom stating that a set is just its elements, not a couple (name, elements).

Also [T. Jech, 2002] tells us that 
"...Newton and Leibniz, do not require the use of infinite sets, and infinity appears only as "a manner of speaking", to paraphrase Friedrich Gauss. The fact that the set of all positive integers has a proper subset, like the set of squares $\{1,4,9,16,25, \ldots\}$ of the same cardinality (using modern terminology) was considered somewhat paradoxical (this had been discussed at length by Galileo among others). Such apparent paradoxes prevented Bernhard Bolzano in 1840s from developing set theory, even though some of his ideas are precursors of Cantor's work. (It should be mentioned that Bolzano, an accomplished mathematician himself, coined the word Menge (= set) that Cantor used for objects of his theory.)",

therefore that a set is only its elements (with the brackets, of course).

The problem is, once more, the difficulty of Russell, or of whoever is attributing this 'doubt' to him, of telling the difference between purely human and mathematical language.

We cannot base ourselves in the concrete world when talking about Mathematics.

We have to accept the world of Mathematics and its limitations instead.

We would only have identified an inconsistency in Set Theory if the axiom that defines sets said that a set is (name, elements) instead of (elements). Since the axiom says precisely the opposite, we actually do not have such a problem.

All Russell had to do was realizing that the set that he created would have to be an 'impossible-to-be-baptized' set, for any name given to it would imply the impossibility of existence of the set since no set can be an element of itself, yet any name he gave the set would have to be inside of it, given the $P(x)$ involved.

In Mathematics, we only have an inconsistency if we get a problem when using the premises that are part of our theory in our inferences. We therefore do not have a problem with this one.

Notwithstanding, we still could argue, in defense of Russell, that the elements, isolated by the curly brackets, should then be inside of the brackets as well since the set, written that way, still does not belong to itself.

The only way out of the paradox is then accepting that the coordinate time is also part of the creation process of a set, even if we do not spell it.

This way, a set is (name, elements, date of creation, time of creation), just like all other entities in human kind.

Because of that,

$R=\{A, B, C, \ldots, Q, S, \ldots, A A, \ldots\}$, with no problems because $R$ is created from the existing sets being tested for $P(x)$, and $R$ does not exist whilst that process is taking place. 
One second after we have selected all sets with the $P(x)$ property, however, $R$ is a set.

If we then forget 'its birth' and see $R$ together with $\{A, B, C, \ldots, Q, S, \ldots, A A, \ldots\}$ and $P(x)$, but not the 'generating process' of $R$, we will think that there was a mistake and $R$ should be inside of the brackets with the other sets.

Notwithstanding, we need to have a description for $R$ and analyze it to prove that $R$ should be inside of the brackets in Mathematics and, in it, all has to be proven.

In going for the 'generating process' for $R$, we will then see that $R$ did not exist when $P(x)$ was tested, what then makes us accept that $R$ be not inside of the brackets with the other sets.

\section{The 'paradox'}

Who saw in the above puzzle a paradox wrote the problem in the following manner:

1) Call normal a set that does not belong to itself.

2) Build $R$, where $R$ is the set of normal sets, that is, of the sets that do not belong to themselves.

3) Notice that if $R$ is told to belong to $R$, then $R$ is normal, therefore $R$ does not belong to $R$, contradiction.

4) Notice that if $R$ does not belong to $R$, then $R$ is not normal, that is, $R$ belongs to $R$, therefore also a contradiction.

5) Since 3 and 4 both lead to absurdities, $R$ cannot exist, therefore inconsistency.

What was the mistake in the lines above?

Just that $R$ can never be imagined to either belong or not belong to $R$ because the set $R$ refers to all sets that exist by the time of its creation, and $R$ itself does not exist by the time of its creation.

\section{On Maple, Mathematics, and time}

So, would that happen to any set that has a $P(x)$ involving testing all sets?

Surely it would.

Example:

$M=\{x / x$ is set that be not empty $\}$. 
$M$ will not be empty, yet $M$ will not belong to $M$ because $M$ does not exist by the time of testing the 'pertinence property' for $M$.

Notice that the same problem does not happen with the relationship of inclusion because, to count the subsets of a set, we imagine the set after it is built in full, always.

Whilst the property $P(x)$ tells who belongs or not to a set, and such a property 'is the own set' somehow, the inclusion relationship is not part of the set, like it is not the own set.

Suppose, however, that we create a set with $P(x)=$ contains itself, would $R$, if defined this way, be an element of $R$ ?

The answer would have to be the same: No, it would not.

Does that mean that we have found the first set that does not contain itself?

No, it does not.

If we consider $R$ formed and we now build $S$ the same way, $R \in S$, that is, $R$ is contained in itself and is a member of $S$, of the sets that contain themselves at date $e / f / g$ and time t:v, say.

Then, one would say, we have the sets $R, S$, and $T$ and we ask for the sets that do not belong to themselves. We then call the set generated this way $R$. Now, we have it 'again' (the paradox)!

The response to such an argument is that $R$ cannot, in Mathematics, be two things at a time, in terms of sets, therefore $R$ cannot be both an element and a set that contains it plus some other elements.

Therefore, it is precisely when we acknowledge time change, that the time of formation of our set of sets is an earlier time than the time of formation of $R$, that we do not have a paradox in Russell's example.

If all things are chosen in the past, we can only judge whether a set belongs to another or not if we know the property that formed the set and the time in which it appeared?

Yes, unfortunately, that is true.

Even though Mathematics simplifies a lot the 'actual life' entities, for instance when it takes away dimensions, as said before, it is not indifferent to time changes, and we then need to acknowledge that formally.

Notice that Maple is a tool for Mathematics, and it does consider time as one of its coordinates.

For instance, if we use the 'attrib' sign of Maple, ': $=^{\prime}$, in the first line of our routine, assigning a value to the variable $x$, say $0(x:=0)$, and we then use $x$ as a variable in a 
few equations, we will have those equations replacing $x$ with 0 in order for Maple to print our result. If we later on use another attrib, say in the tenth line of our routine, assigning another value to the variable $x$, say $1(x:=1)$, and we then use $x$ as a variable in a few equations after that, we will have those equations replacing $x$ with 1 in order for Maple to print our result.

Therefore, Maple reads $x$ from the last assignment and 'knows' which one is the last assignment. If we were to explain what is happening in the computer's mind by the time that happens, we could then say that the computer took note of the first assignment by writing $x(t=0)=0$ and took note of the last assignment by writing $x(t=0.5)=1$, that is, the computer counted the time of each assignment.

Mathematics, unfortunately, is way behind the computers then, since it is not acknowledging time change in 'its brains'. That is then our work here and in at least another paper of ours (regarding the Cartesian Plane): Acknowledging time change formally in Mathematics.

If time change is finally acknowledged formally by Mathematics, then Russell does not have a paradox.

Notice that after a set goes through the process of 'breaking water', let's say, then it becomes a name and its elements, and the rule should not be ever applied again, like the $P(x)$ s, let's say, are supposed to generate at most one kid, and what now exists is $M=\{R, S, T\}$, say, not $M=\{x / x$ is a set that contains itself in the 'universe $w$ ' $\}$.

Also notice that we always need limitations in Mathematics, specifications, so that a set is formed with basis in a certain 'universe', that is, it is a relative thing, not absolute.

The universe, in the case of Russell's set, should be understood as being 'sets that are fully formed before the time of application of "the rule" (this, per se, will force the readers to acknowledge 'time change').

\section{The paradox in its original version}

In The Principles of Mathematics, a publication of 1903 [Bertrand Russell, 1903], Bertrand Russell had, in Appendix B. The Doctrine of Types, section 500, what would be nowadays re-worded and called Russell's Paradox (see [K. C. Klement, 2001]):

"...for we can prove that the logical product of $w$ both is and is not a member of $w$. This seems to show that there is no such range as $w$; but the doctrine of types does not show why there is no such range. It seems to follow that the Contradiction requires further subtleties for its solution; but what these are, I am at a loss to imagine."

Russell had arrived at such a conclusion following the reasoning of Cantor when proving that the irrational numbers are not countable. 
In his book, he assumes that there is a particular one-one relation, "which associates every proposition $p$ which is not a logical product with the range whose only member is $p$, while it associates the product of all propositions with the null-range of propositions, and associates every other logical product of propositions with the range of its own factors. Then the range $w$ which, by the general principle of Cantor's proof, is not correlated with any proposition, is the range of propositions which are logical products, but are not themselves factors of themselves. But, by the definition of the correlating relation, $w$ ought to be correlated with the logical product of $w . "$

Already here we notice that there is something wrong with the piece of theory Russell now uses to criticize his own theories.

Notice that what he writes would imply at least writing a list of logical propositions in terms of codes and then forming a new proposition using the 'diagonal reasoning', which is what Cantor is told to have done.

The problem is that, just like Cantor, we will then be guaranteeing that we obtain, in this way, a new element, which will be a new logical proposition, not yet in our list.

Because it will be formed by a piece of each element in the list, diagonally picked, in a fashion that the $n^{\text {th }}$ digit of the new number, formed this way, will be different from the $n^{\text {th }}$ digit of the element in the $n^{\text {th }}$ position in the list, it should be different from all others, but Russell, different from Cantor, does not let us know how he will be writing his list, so that we could have propositions that vary only in the last piece of the code, let's say, what would then make it possible that we pick the same proposition again.

Different from Cantor's elements, our propositions and our logical products are finite things, so how could Russell ever think that Cantor's Diagonal applied to them?

We write about this matter in detail in the next section, but Russell then 'explains':

"If $m$ be a class of propositions, the proposition "every $m$ is true" may or may not be itself an $m$. But there is a one-one relation of this proposition to $m$ : if $n$ be different from $m$, "every $n$ is true" is not the same proposition as "every $m$ is true". Consider now the whole class of propositions of the form "every $m$ is true", and having the property of not being members of their respective $m$ 's. Let this class be $w$, and let $p$ be the proposition "every $w$ is true". If $p$ is a $w$, it must possess the defining property of $w$; but this property demands that $p$ should not be a $w$. On the other hand, if $p$ be not a $w$, then $p$ does possess the defining property of $w$, and therefore is a $w$. Thus the contradiction appears unavoidable."

What we understand here is that $m$ is a set of propositions. If we create a proposition, say "every $m$ is true", then this proposition, which we have just created, may belong to $m$ or not. 
However, we can always create a one-to-one relation of the proposition we have just created to $m$ : If $n$ be different from $m$, "every $n$ is true" is not the same proposition as "every $m$ is true".

Now, we create a set of propositions, which we call $w$, with all propositions of the type "every $m$ is true" that have the property of not belonging to $m$, that particular set of propositions of the beginning of this sequence of lines.

Make $p=$ "every $w$ is true".

If $p$ is a $w$, that means that $m=w$ as well and $p$ does not belong to $w$, what is then $a$ contradiction.

If $p$ is not a $w$, that means that $p$ belongs to the set $w$, therefore contradiction.

If one reads the reasoning in italics, one really gets confused and thinks that Russell really found some problem with his own theories.

However, if we detach ourselves from what is written in italics, like if we let the emotions vanish, we notice that the supposed set $w$ could never exist when $m=w$ because we cannot form a set from elements that 'cannot be in it'.

We had

"Now, we create a set of propositions, which we call w, with all propositions of the type "every $m$ is true" that have the property of not belonging to $m$, that particular set of propositions of the beginning of this sequence of lines."

Now, replacing $m$ with $w$, we get:

Now, we create a set of propositions, which we call $w$, with all propositions of the type "every $w$ is true" that have the property of not belonging to $w$, that particular set of propositions of the beginning of this sequence of lines.

Notice as well that Russell calls both the individual propositions and the set of those $w$ to get his example, but, as we know, $w$ could never be both at the same time.

Russell also tries to explain 'the problem' in another way:

"If $m$ be a class of propositions, their logical product is the proposition "every $m$ is true", which I shall denote by ^ ' $m$. If we now consider the logical product of the class of propositions composed of $m$ together with $\wedge^{\wedge} m$, this is equivalent to "Every $m$ is true and every $m$ is true", i.e., to ^ $m$. Thus the logical product of the new class of propositions is equivalent to a member of the new class, which is the same as the logical product of $m$. Thus if we identify equivalent propositional functions ( $\wedge^{`} m$ being a propositional function of $m$ ), the proof of the above contradiction fails, since every proposition of the form $\wedge^{\wedge} m$ is the logical product both of a class of which it is a member and of a class of which it is not a member." 
This example sounds even more complex, but is based on the creation of a class that is the 'square', let's say, of another class, and this square coincides with the class that is squared in terms of results.

The core of the argument is that the result, the squared class, is equal to the own class, but we got to the second factor being the own class by multiplying logically the original class by its logical product, what then means that if $p$, a proposition, belongs to the square of the original class, then it belongs to its own class and to the logical product of it. If we replace 'every $w$ is true', by the moment of deciding on the pertinence to $w$ of $p$, with this 'equivalent expression' that we found, then there is no paradox because $p$ will not belong to $w$, as needed.

Notice that, in replacing the expression with its equivalent, Russell actually got rid of the main problem, which is that the class would be formed by the elements that cannot be in it. With the replacement, the class is formed by the elements of another class, which is not part of the unacceptable restriction.

A class that is formed exclusively by the elements that cannot be in it is not a proper class, cannot exist.

This is the same as saying put the liquid that cannot be in plastic in that plastic container.

Basically, our set, this way, 'blows up', just like the plastic container in the justdescribed situation.

\section{Russell's mention to Cantor's Diagonal}

Notice that

"there is a particular one-one relation, 'which associates every proposition $p$ which is not a logical product with the range whose only member is $p$, while it associates the product of all propositions with the null-range of propositions, and associates every other logical product of propositions with the range of its own factors. Then the range $w$ which, by the general principle of Cantor's proof, is not correlated with any proposition, is the range of propositions which are logical products, but are not themselves factors of themselves. But, by the definition of the correlating relation, $w$ ought to be correlated with the logical product of $w .^{\prime}$ "

confounds us quite a lot. Basically, we should be able to express any proposition as a result of a logical operation of itself and another proposition for, if nothing else, we can always say that $\mathrm{AA}=\mathrm{A}$.

If we do not want our proposition to be a factor of itself, then we do not want trivial operations, right? 
Notwithstanding, it has to be true that all propositions can be written in the shape of a logical product, so what is he actually saying in the first lines of this paragraph?

He says 'associates every proposition $p$ which is not a logical product with the range whose only member is $p^{\prime}$.

Ah, so he means that we consider all propositions to 'pre-exist', let's say, and we select only those that do not have the shape of a logical product for this step.

At the end of the paragraph, we will take those that are logical products, but products where they are not one of the factors.

Right.

Now, we understand that $w$ is a 'new class', not yet mentioned/created, even though it clearly be associated to a subclass of the class 'own factors', let's say.

Russell then asserts that this new class is not related to any proposition, and that must be because he is using his own example (he uses the same name, $w .$. ), since it is almost impossible to correlate his new situation with Cantor's Diagonal.

We cannot have any element in the class because otherwise we get contradiction.

However, for being a logical product involving $w$, the proposition must be associated with the range containing $w$.

It is just that if we do not have any elements in this class, there will be no association and we can obviously always create 'impossible sets' ... .

In this case, there is no possible association, therefore this proposition has not been listed yet, or correlated, yet all of 'that type' have (and that is why he mentions Cantor)... . Well, there is no proposition here... . Cantor exhibited an actual number instead: Very different... . It is all inappropriate, then.

That is when Russell says 'contradiction', basically.

The problem with this reasoning is obvious.

As said before, Cantor had, with his diagonal, guaranteed that his $w$ were different from every other member of the list by at least one digit.

Russell, on the other hand, gets 'domain elements' that already have 'images' assigned to them, therefore that already have been listed, and those have been listed in full.

There is obviously no possible parallel here.

This was just an improper reading of Cantor's argument by Russell... .

Notice as well that Russell equates a range called $w$ with a range containing $w$ to get his result. 


\section{Conclusion}

Russell's Paradox is one more allurement, this time in Mathematics. Russell had a doubt that he passed to Frege. Frege did not notice the doubt of Russell and assumed that Russell's doubt was the proof of him (Frege) being wrong in something. Frege perhaps was in a rush because his second volume was being printed or perhaps he trusted Russell a 'bit too much'. Basically, R, the set of the 'normal' sets, is not a proof of inconsistency of the known axioms of Mathematics if we acknowledge the existence of the coordinate time in the creation of the mathematical objects.

Russell's Paradox only appears due to distraction of those who had contact with it this far.

We do include the coordinate 'time' when 'playing with Mathematics' in practice, but we need to acknowledge its existence in the mathematical writings to make its theories acceptable.

Basically, every set is built with basis in a special 'universe', defined either implicitly or explicitly through their 'formation property', which we have called $P(x)$ here.

Since there is a clear parallel between purely human and mathematical languages, we could use the figure of discourse called ellipsis to explain our 'solution'.

As we know, we are 'allowed' to use ellipsis in purely human language, and we tend to think that using them makes our discourse 'more elegant'.

Notwithstanding, Mathematics aims for maximum clarity and quickest plus most accurate understanding of the message, being in that very different from the purely human language, therefore we should probably spell out our 'universes of reference' when we are spelling our $P(x)$ s. That will finish with 'Russell's examples' by the time of creation of those.

On the other hand, it is all common sense, so perhaps we really do not need to do that since we also do not spell, for instance, 'even numbers from amongst the nonnegative integers', but nobody has come up with a 'Russell's example' to prove our theories of set formation to be inconsistent there, what means that they all accept that the 'universe of reference' for 'even numbers' is the 'non-negative integers'. 


\section{Bibliography}

A. D. Irvine. (2010). Bertrand Russell. The Stanford Encyclopedia of Philosophy (Winter 2010 Edition), Edward N. Zalta (ed.). Retrieved November 72011 from http://plato.stanford.edu/archives/win2010/entries/russell/.

A. D. Irvine. (2009). Russell's Paradox. The Stanford Encyclopedia of Philosophy (Summer 2009 Edition), Edward N. Zalta (ed.). Retrieved November 72011 from http://plato.stanford.edu/archives/sum2009/entries/russell-paradox/.

B. Russell. (1903). The Principles of Mathematics. Retrieved September 302012 from http:// fair-use.org/bertrand-russell/the-principle-of-mathematics.

Eric W. Weisstein. (1999). Subset. MathWorld-- A Wolfram Web Resource. Retrieved November 72011 from http:// mathworld.wolfram.com/Subset.html.

K. C. Klement. (2001). Russell's Paradox in Appendix B of the Principles of Mathematics: Was Frege's response adequate? History and Philosophy of Logic, vol. 22, pp. 13-28.

Stephan Körner. (1986). The philosophy of Mathematics: An introductory essay. Courier Doves Publications. ISBN-10: 0486250482, ISBN-13: 9780486250489.

Thomas Jech. (2002). Set Theory. The Stanford Encyclopedia of Philosophy (Summer 2009 Edition), Edward N. Zalta (ed.). Retrieved October 32011 from http://stanford.library.usyd.edu.au/entries/set-theory/\#1.

Wolfram Team. (1999). Russell's Antinomy. MathWorld-- A Wolfram Web Resource. $\begin{array}{llll}\text { Retrieved } & \text { October } & 2 & 2012\end{array}$ http://mathworld.wolfram.com/RussellsAntinomy.html. ${ }^{*}$ 


\section{E-LOGOS}

ELECTRONIC JOURNAL FOR PHILOSOPHY

Ročník/Year: 2012 (vychází průběžně/ published continuously)

Místo vydání/Place of edition: Praha

ISSN 1211-0442

Vydává/Publisher:

Vysoká škola ekonomická v Praze / University of Economics, Prague

nám. W. Churchilla 4

Czech Republic

13067 Praha 3

IČ: 61384399

Web: http://e-logos.vse.cz

Redakce a technické informace/Editorial staff and technical information:

Miroslav Vacura

vacuram@vse.cz

Redakční rada/Board of editors:

Ladislav Benyovszky (FHS UK Praha, Czech Republic)

Ivan Blecha (FF UP Olomouc, Czech Republic)

Martin Hemelík (VŠP Jihlava, Czech Republic)

Angelo Marocco (Pontifical Athenaeum Regina Apostolorum, Rome, Italy)

Jozef Kelemen (FPF SU Opava, Czech Republic)

Daniel Kroupa (ZU Plzeň, Czech Republic)

Vladimír Kvasnička (FITT STU Bratislava, Slovak Republic)

Jaroslav Novotný (FHS UK Praha, Czech Republic)

Jakub Novotný (VŠP Jihlava, Czech Republic)

Ján Pavlík (editor-in-chief) (VŠE Praha, Czech Republic)

Karel Pstružina (VŠE Praha, Czech Republic)

Miroslav Vacura (executive editor) (VŠE Praha, Czech Republic) 